\title{
Comparison of Organ Location, Morphology, and Rib Coverage of a Midsized Male in the Supine and Seated Positions
}

\author{
Ashley R. Hayes, ${ }^{1,2}$ F. Scott Gayzik, ${ }^{1,2}$ Daniel P. Moreno, \\ R. Shayn Martin, ${ }^{2}$ and Joel D. Stitzel ${ }^{1,2}$ \\ ${ }^{1}$ Virginia Tech, Wake Forest University Center for Injury Biomechanics, 575 N. Patterson Avenue Suite 120, \\ Winston-Salem, NC 27101, USA \\ ${ }^{2}$ Wake Forest University School of Medicine, Medical Center Boulevard, Winston-Salem, NC 27157, USA
}

Correspondence should be addressed to F. Scott Gayzik; sgayzik@wakehealth.edu

Received 5 December 2012; Revised 15 February 2013; Accepted 21 February 2013

Academic Editor: Costin D. Untaroiu

Copyright (C) 2013 Ashley R. Hayes et al. This is an open access article distributed under the Creative Commons Attribution License, which permits unrestricted use, distribution, and reproduction in any medium, provided the original work is properly cited.

\begin{abstract}
The location and morphology of abdominal organs due to postural changes have implications in the prediction of trauma via computational models. The purpose of this study is to use data from a multimodality image set to devise a method for examining changes in organ location, morphology, and rib coverage from the supine to seated postures. Medical images of a male volunteer $(78.6 \pm 0.77 \mathrm{~kg}, 175 \mathrm{~cm})$ in three modalities (Computed Tomography, Magnetic Resonance Imaging (MRI), and Upright MRI) were used. Through image segmentation and registration, an analysis between organs in each posture was conducted. For the organs analyzed (liver, spleen, and kidneys), location was found to vary between postures. Increases in rib coverage from the supine to seated posture were observed for the liver, with a $9.6 \%$ increase in a lateral projection and a $4.6 \%$ increase in a frontal projection. Rib coverage area was found to increase $11.7 \%$ for the spleen. Morphological changes in the organs were also observed. The liver expanded 7.8\% cranially and compressed 3.4\% and 5.2\% in the anterior-posterior and medial-lateral directions, respectively. Similar trends were observed in the spleen and kidneys. These findings indicate that the posture of the subject has implications in computational human body model development.
\end{abstract}

\section{Introduction}

The fatalities and injuries associated with motor vehicle crash remain a leading problem in the United States. In 2010, the National Highway Traffic Safety Administration reported 30,196 individuals were killed in 5.4 million police-reported automobile crashes. In addition roughly 1.5 million people were injured in these police-reported motor vehicle crashes [1]. The University of Michigan Transportation Research Institute used the National Automotive Sampling System (NASS) to show approximately 19,000 adult occupants sustain abdominal injuries per year. Of those 19,000 adult occupants, the liver and spleen are found to be the most commonly injured organs in frontal impact cases, with kidney injury being minimal [2]. These injuries often result from loads from the steering wheel, seat belt, or other interior features of a vehicle, such as the door in a near-side lateral impact, and the literature has reported that rib fracture can lead to damage of abdominal organs including the liver, spleen, and kidneys [36]. Additionally, literature reported that low right-sided rib fracture increased the probability of liver injury, and low leftsided rib fracture increased the probability of spleen injury [3-6]. Al-Hassani et al. also concluded lower rib fracture result in kidney injury [5].

Abdominal injuries are not exclusive to the civilian population. Military personnel are also susceptible to nonpenetrating ballistic and blast impact which lead to internal abdominal injuries, such as liver laceration [7]. In blast events, air-containing organs including the lungs, larynx, trachea, and gastrointestinal region are the primary sources of injury, followed by solid organs like the liver, spleen, and kidneys. Although these abdominal organs are not as vulnerable to blast injury as air-containing organs, lacerations or lethal hemorrhage can occur [8]. 
Given these statistics regarding injury and fatalities associated with vehicle crashes, ballistic impact, and blast exposure, models of the human body (including physical surrogates and computational models) have been developed. These models are used to investigate injury mechanisms, evaluate safety system designs, and inform physical testing prior to conducting experiments. Finite element (FE) computer models are often used by researchers to examine the biomechanical response of the human body in blunt injury scenarios such as vehicle crash [9-15]. When examining the medical image data sets used in the development of various full human body computational models, typically only one image modality or one posture (supine) is used to develop the model's geometry. This is true for several models currently found in the literature $[10,16,17]$.

Often the organ geometry that is used to create these finite element models is based on cadaveric organ position, serial sectioning, or the imaging of a subject's organs in the supine position. These methods do not consider the effects of gravity on the location or morphology of the soft tissues within body, as they would be in the seated vehicle occupant position. The morphology and location of thoracoabdominal organs relative to surrounding bony structures in a given posture are likely to play a role in the predicted injury severity. More realistic representation of bone and organ position would allow for a greater ability of models to predict injury based on given load path or component contacted. Physical surrogate models are also used by researchers to examine injury, specifically injury from ballistic impact $[18,19]$. The location and morphology changes of abdominal organs due to specific postures may have implications for the prediction of injuries using these models.

The purpose of this study was to use a three-dimensional dataset of the human body to quantify abdominal organ location and rib coverage based on postural changes in an individual representing the 50th percentile male.

The literature contains only limited studies on the effects of organ position and morphology as a result of postural changes. Recently, Beillas et al. [20] and Lafon et al. [21] examined morphology and location changes of thoracic and abdominal bones and soft tissues using one image modality, Upright Magnetic Resonance Imaging (uMRI). Bony landmarks, the kidneys, liver, spleen, the abdominal cavity, and the thoracic cavity were all examined in four different postures: standing, supine, seated, and forward flexed. The study found that organ volume was mostly unaffected by posture, and abdominal organ location between standing, seated, and the forward flexed position was minimal. When comparing these three postures to the supine posture, the position of solid organs changed by as much as $40 \mathrm{~mm}$. Additionally, studies concluded that abdominal geometry should be corrected for organ movement when using the supine position for finite element modeling [20, 21].

Beillas' work, however, was limited to a single imaging modality, Upright MRI. At 0.6 Tesla, the MRI used to acquire these images (Fonar Upright MRI, Melville, NY) provides less than half the magnetic field strength of a conventional closed bore MRI. The current study builds on these findings by including data from the same model Upright MRI scanner used by Beillas et al., but additionally used Computed Tomography (CT) and $1.5 \mathrm{~T}$, closed bore, MRI data [22]. CT scans provided patient specific bone geometry, so that the relative locations of bones to the organs of interest in both postures could be investigated. Using this data, rib coverage for the liver, spleen, and kidneys in the supine and seated positions was calculated.

\section{Methods}

The subject and imaging protocol was approved by the Wake Forest School of Medicine Institutional Review Board (IRB, number 5705) [22]. Computed Tomography (CT) and Magnetic Resonance Imaging (MRI) scans were used to study the effect of the seated and supine postures on the rib coverage, location, and morphology of abdominal organs. The supine posture is representative of the conventional MRI or CT with the patient lying on their back, while the seated posture employed is representative of a vehicle occupant [2325]. A single subject, targeted to represent the 50th percentile male (M50) in terms of height and weight $(174.9 \mathrm{~cm}$ and 78.6 $\pm .77 \mathrm{~kg}$ ) [25] and other anthropometric targets, was scanned in three image modalities [22]. The complete data set contains over 15 thousand individual images from this individual.

Given the volume of image data to be collected in the protocol and project objectives, selection of a single volunteer meeting numerous anthropometric criteria was favored over a larger study population. The comprehensive 1988 Anthropometric Survey of United States Army Personnel (ANSUR) study was used as the basis for the selection criteria [26]. The volunteer met 15 target anthropometric criteria for the 50th percentile male within an average deviation of 3\% [25]. The subject volunteer was screened for basic health data, which included a clear medical history with no history of osteopenia or osteoporosis, claustrophobia, metal implants, major surgeries involving organ removal, or any implanted electrical devices [22]. The subject's anatomy was reviewed by a collaborating radiologist and was deemed to be free of anatomical abnormalities or pathology.

The main goal of this study was to compare the change in organ location, rib coverage, and morphology in the seated and supine postures. To accomplish this, six steps were conducted. The first two concerned image acquisition and composition, which is the method for combining several image sets into one continuous image set. Following this, the data were extracted through segmentation, analyzed for organ exposure variations, aligned in a common coordinate system, and analyzed for gross location relative to the Center of Gravity (CG). Finally, each organ segmented from the seated posture was independently aligned to its counterpart model in the supine posture to analyze morphological variations between postures. Each of these steps is described in greater detail in the following.

2.1. Image Acquisition. Image acquisition are described in detail in previous publications [22] but are reviewed here briefly. Supine MRI image resolution was $0.78 \mathrm{~mm}$, slice thickness was $2 \mathrm{~mm}$ depending on the region, and the field of 


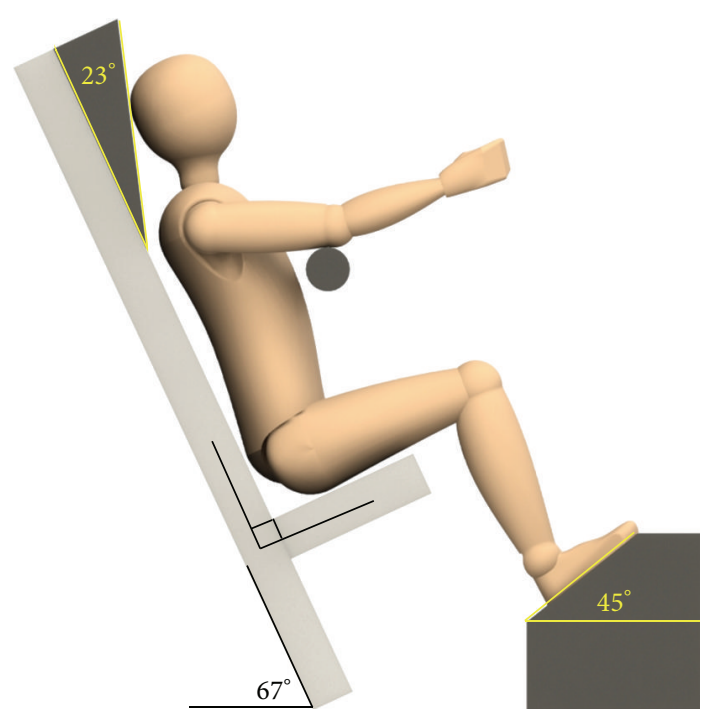

(a)

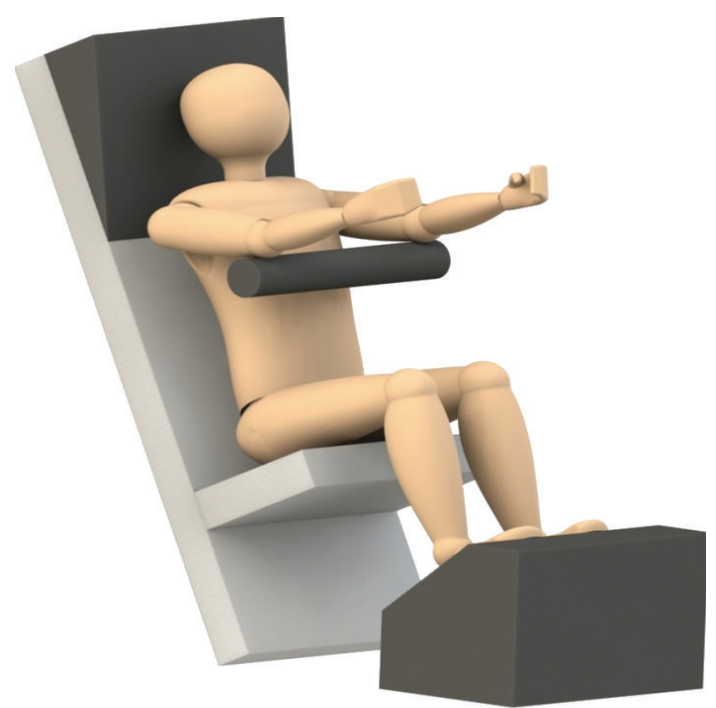

(b)

FIGURE 1: Sketches of seated scan position.

view (FOV) for the supine MRI images was $400 \mathrm{~mm}$. Seated MRI image resolution was $2.1 \mathrm{~mm}$, image slice thickness ranged from 1.5 to $2 \mathrm{~mm}$, and the FOV was $430 \mathrm{~mm}$. Supine CT images had a resolution of $0.40 \mathrm{~mm}$, image slice thickness of $0.63 \mathrm{~mm}$, and FOV of $500 \mathrm{~mm}$.

The seated position used for the collection and analysis of data is shown in Figure 1. For the supine position the subject was lying on a table in the horizontal position, and for the seated position the seat back angle was placed at 23 degrees from vertical, and the thigh angle from horizontal was recorded for consistency between image acquisitions [22]. The seated position was chosen based on data from the literature [27].

2.2. Image Composition. The supine MRI and seated uMRI scans were combined using five (supine) and six (seated) image sets, ranging from the neck to the pelvis. The software program Amira (Visual Imaging Inc, San Diego, CA) was used to combine the separately acquired image sets into contiguous data sets (one for supine and one for seated). These data sets were then used for organ segmentation and bone placement.

2.3. Image Segmentation. The vertebral bodies (T5 through L5), sacrum, pelvis, sternum, and ribs 5 through 12 were segmented in the CT supine scan (threshold: min 226, max 1940) and were then repositioned and registered into the MRI supine and uMRI seated scans using Mimics (v. 14, Materialise, Leuven, Belgium). The bones initially were manually repositioned through translation and rotation in the anteriorposterior, right-left, and cranial-caudal directions. For finer adjustment, a point-based registration technique was used. In this approach, approximately 50 points were selected on the bone and the corresponding location on the MRI and uMRI scans, and the two sets of points were registered in space.
Only vertebral bodies T5 through L5 and ribs 5 through 12 were used due to their proximity to the abdominal organs of interest.

The liver, spleen, and kidneys were then manually segmented in the supine MRI scan to create mask for each respective organ (Figure 2). Masks are groupings of pixels meant to represent structures of interest. A 3-dimensional (3D) model was created using each abdominal organs mask (Figure 2) and was imported into Geomagic Studio (v. 11, Geomagic, Raleigh, NC) software to refine the polygon model exported by Mimics. The refining process included spike removal, defeaturing (a smoothing process), and filling holes in the surface model.

In the uMRI scan, the 3D models that were created in the MRI supine position were imported and repositioned. Once the 3D model of each abdominal organ was positioned manually in the uMRI modality, a mask was created from each 3D model. Due to changes in the posture, manual editing of the masks was then required to complete each abdominal organs mask, and 3D models were created. Per the same process outlined previously for the supine MRI data, each 3D model was created, and imported into Geomagic Studio for a similar refining process. A final visual inspection of the contours of each mask was conducted to ensure good agreement with the scan data.

2.4. Quantification of Rib Coverage. The computer-aided design (CAD) software package, Rhinoceros (v4.0, McNeel and Associates, Seattle, WA), was used to quantify rib coverage. In this study, we define a term organ rib coverage to mean the area of an organ directly deep to the ribs in an anterior, lateral, and/or posterior projection. The study investigated the projection of ribs 5 through 12 onto the abdominal organs of interest. Anterior, left, posterior, and right posterior-lateral were views defined to examine rib 


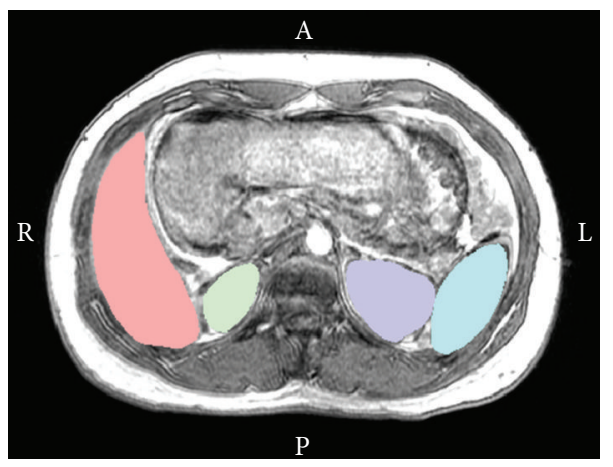

(a)

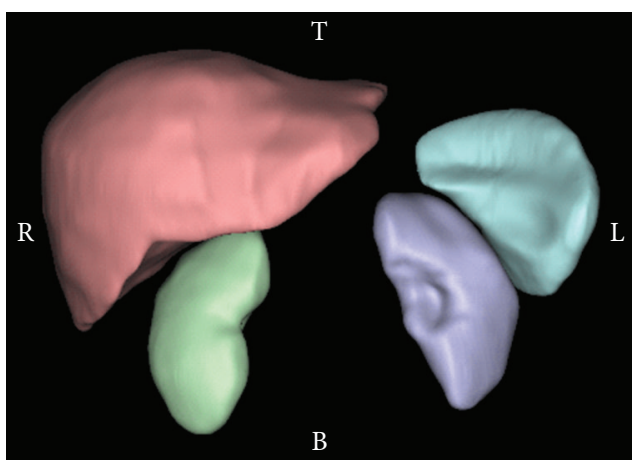

(b)

Figure 2: Manual segmentation of abdominal organs (a) and 3D model of abdominal organs (b) in the supine position. A: anterior, P: posterior, R: right, L: left, T: top, and B: bottom.

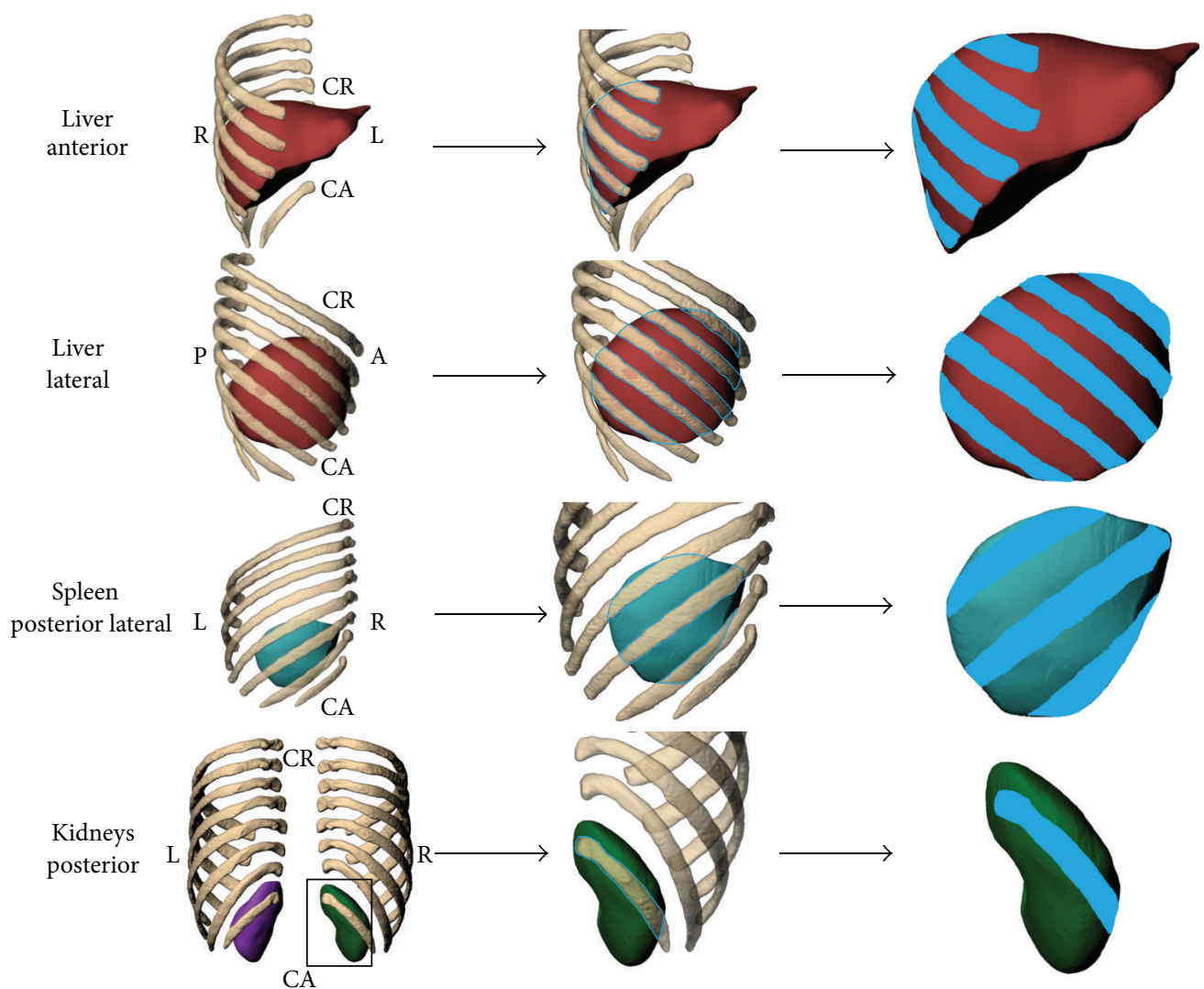

FIGURE 3: Views and method for estimating rib coverage. Note that the supine organs are shown, and the process was repeated for organs in the upright scan as well. Totals for the bone projected area are provided in the results.

coverage (Figure 3). Depending on the proximity of the organ to the ribs, one or more projections were conducted. The liver was analyzed with anterior and lateral projections, the spleen with a single right posterolateral projection, and the kidneys with a single posterior projection.

Within each respective view, a polyline was used to define the outline of rib coverage on the organ of interest and to define the total organ area of the projected view (Figure 3). One projected view was calculated for each organ, except for the liver, where both anterior and lateral projections were made. The polylines created were imported into Geomagic Studio, along with the models of the abdominal organ of interest. The polylines that represented rib coverage were projected onto the surface of the respective abdominal organ. The projected area of the ribs on the organ can be seen in Figure 3. The surface area was summed for all ribs in each view of interest. The percent area of rib coverage was calculated for the four abdominal organs of interest, in both 


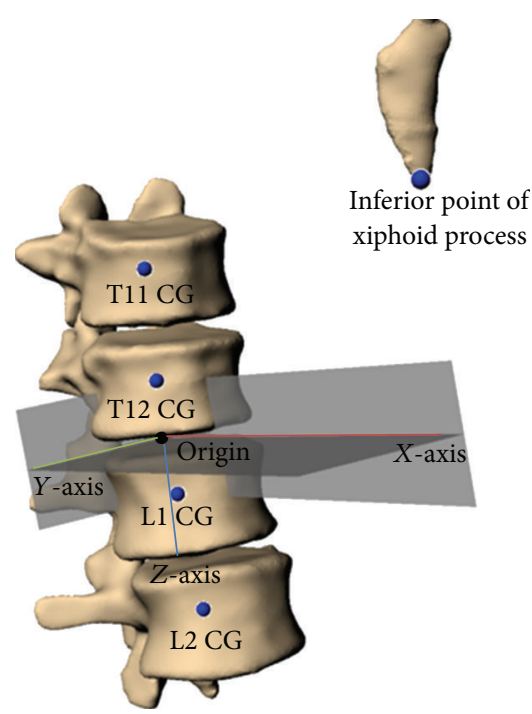

FIGURE 4: Local coordinate system defined for CG relative location analysis.

the supine and seated positions, by subtracting the organ exposure from the total surface area in the projected view and dividing that value from the project view total surface area.

2.5. Data Alignment. At this stage in the study, two separate models of the thoracolumbar spine, ribs 5-12, and selected abdominal organs were complete: one in the supine and one in the seated posture. The next step was to align these two models into the same space to analyze differences in position. Vertebral bodies T11 through L2 were selected as the base structures for alignment due to similar curvature of the spine in this region between each data set. These four structures in the supine MRI scan were selected as the reference set. The transformation matrix to move only T11 through L2 from the seated to the supine posture was determined using Geomagic Studio. This transformation matrix was then applied to all uMRI structures. Note that this was a rigid transformation that preserved the relative distance between all bony landmarks and organs of upright scan structures.

With the two segmented data sets now within a common space, a comparative analysis of abdominal soft tissue relative location was conducted. To quantitatively examine organ relative location, a local coordinate system was defined (Figure 4). The coordinate system was defined loosely based on the work by Wu et al. [28] but modified to align with the SAE J211 coordinate system [29]. The positive $X$ was defined to be anterior, positive $Y$ was defined to the right, and positive $Z$ was defined to be downward. The origin was set as the midpoint between the CG of T12 and L1. The $X-Z$ plane was defined using three points: the origin, the CG of L2, and the most inferior point of the xiphoid process. The $z$-axis was defined as the line passing through origin and the CG of L2. The $x$-axis was defined as the line in the $X-Z$ plane perpendicular to the $z$-axis. Using the right-hand rule, the $y$-axis was defined to be orthogonal to the $x$ - and $z$-axes. The center of gravity locations described in the following was taken with respect to this local coordinate system, and differences between these landmarks were measured.

2.6. Morphology Comparison. Finally, each abdominal organ in the supine and seated positions was imported into a common space and aligned to the respective organ in the supine position. This second alignment was needed to examine morphology changes in each organ from the supine to seated position, while the previous alignment using the vertebral column examined gross abdominal organ movement between postures. The seated organ was aligned to the supine organ using Geomagic Studio software, and a sample size of 10,000 points was used with a tolerance of $0.1 \mathrm{~mm}$. The sample size defines a number of points on the surface of each object of interest that are chosen in an attempt to ensure that a full range of normal directions are being represented in the sample. The tolerance is the degree of allowable error that will be used during the alignment process. After selecting the number of points to use in the alignment, the points are randomly distributed on the surface. Deviations were calculated by first selecting a reference object and a test object. The reference object was always the respective organ in the supine position, and the test object was always the organ in the seated position. Deviations are then reported as the shortest linear distance from the test object to any point on the reference object and therefore quantify organ surface topology changes between postures. The deviation analysis reports the largest positive and negative distances from the test object to any point on the reference object. Deviation values are identified on the reference object at the points of the polygonal surface. Positive values of the deviation analysis indicate expansion of the organ in the region, while negative values indicate compression. A bounding box was created around the $3 \mathrm{D}$ models of the liver, spleen, and kidneys in the supine and seated positions, and the dimensions were noted. This bounding box was created for all models in the local coordinate system described previously. The difference in dimensions of the bounding box along each orthogonal direction was used to provide normalized values of organ morphology changes in addition to the surface deviations.

\section{Results}

3.1. Rib Coverage. Rib coverage, based on variations in rib location for each abdominal organ in the supine and seated positions, was quantified based on methods defined previously (Figure 5). Larger variations of coverage were observed for the liver, spleen, and right kidney, with minimal differences of rib coverage for the left kidney. Through visual examination, the ribs are shown to rotate anteriorly and superiorly from the supine to seated position creating a large area of rib coverage for the liver and spleen in the seated position (Figure 3). The area of rib coverage for the liver was found to increase $4.6 \%$ in the anterior view and $9.6 \%$ in the lateral view. The area of coverage for the spleen increased $11.7 \%$, whereas the right and left kidneys had a decrease in rib coverage of $12.2 \%$ and $0.4 \%$ when transitioning to the seated position (Table 1). 
TABLE 1: Area organ exposure and rib coverage for abdominal organs in the supine and seated positions.

\begin{tabular}{|c|c|c|c|c|c|c|c|}
\hline \multirow[b]{2}{*}{ Organ } & \multicolumn{3}{|c|}{ Supine, $\mathrm{mm}^{2}$} & \multicolumn{3}{|c|}{ Seated, $\mathrm{mm}^{2}$} & \multirow[b]{2}{*}{ Difference, \% } \\
\hline & $\begin{array}{c}\text { Total surface } \\
\text { area }\end{array}$ & $\begin{array}{c}\text { Organ } \\
\text { exposure area }\end{array}$ & $\begin{array}{c}\text { Area covered, } \\
\%\end{array}$ & $\begin{array}{c}\text { Total surface } \\
\text { area }\end{array}$ & $\begin{array}{c}\text { Organ } \\
\text { exposure area }\end{array}$ & $\begin{array}{c}\text { Area covered, } \\
\%\end{array}$ & \\
\hline Left kidney posterior & 7424 & 6014 & 19.0 & 7869 & 6409 & 18.6 & 0.4 \\
\hline Right kidney posterior & 7395 & 5775 & 21.9 & 7436 & 6712 & 9.7 & 12.2 \\
\hline Spleen lateral posterior & 10012 & 4901 & 51.1 & 9945 & 3703 & 62.8 & -11.7 \\
\hline Liver anterior & 27806 & 19859 & 28.6 & 25648 & 17140 & 33.2 & -4.6 \\
\hline Liver lateral & 26993 & 14073 & 47.9 & 25817 & 10976 & 57.5 & -9.6 \\
\hline
\end{tabular}

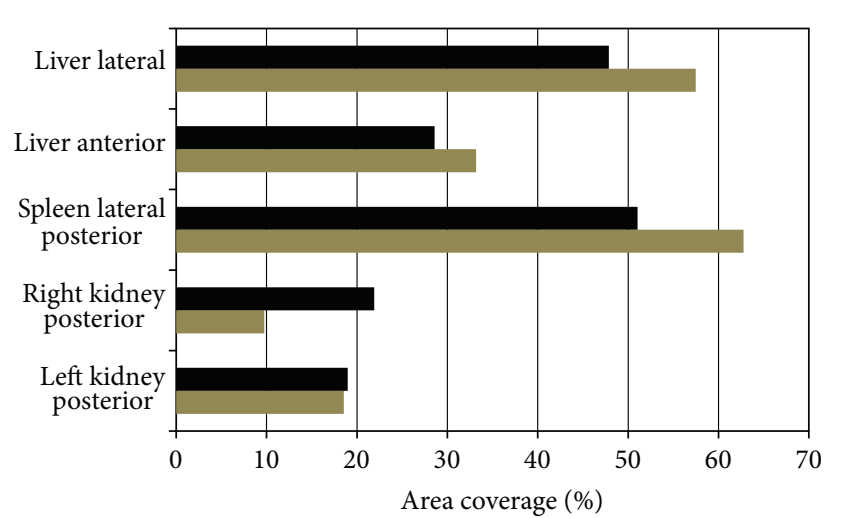

$$
\begin{aligned}
& \text { - Supine } \\
& \text { - Seated }
\end{aligned}
$$

FIGURE 5: Area of rib coverage for the liver, spleen, and kidneys from the supine to seated position.

3.2. Organ Location. Organ location was first examined qualitatively with the organs and bones from the supine and seated scans in the same space. Recall that the vertebral bodies T11 through L2 were used to align the supine and seated sets. The average error from this vertebral body alignment was $0.54 \mathrm{~mm}$. Minimal translation was seen in the mediallateral and anterior-posterior directions for the liver, with the greatest translation being in the cranial-caudal direction (Figure 6). A similar response was seen for the right kidney, except the greatest translation was in the anterior-posterior directions. The spleen was found to translate mostly in the medial-lateral and cranial-caudal directions, while minimal translation of the left kidney was observed (Figure 6).

Distances from the local coordinate system origin to the CG of each abdominal organ of interest were measured in the cranial-caudal, medial-lateral, and anterior-posterior directions for the seated and supine positions to quantitatively examine translation (Table 2 ). Table 2 provides center of gravity translation from the supine to seated position with $X$ being positive anterior, $Y$ being positive left, and $Z$ being positive downward. The liver was found to have large cranial-caudal $(19.5 \mathrm{~mm})$ and medial-lateral translations $(10.0 \mathrm{~mm})$ (Table 2$)$. Therefore, the liver CG translated interiorly and towards the midline. For the spleen, there was greater translation in the medial-lateral and cranial-caudal directions (12.0 $\mathrm{mm}$ lateral and $13.3 \mathrm{~mm}$ cranial) than in the
TABLE 2: Measurements of CG translation from the supine to seated position using defined coordinate system, dimensions in $\mathrm{mm}$.

\begin{tabular}{lcccc}
\hline Organ & $\begin{array}{c}\Delta X \\
\text { Posterior- } \\
\text { anterior } \\
(+)\end{array}$ & $\begin{array}{c}\text { Left-right } \\
(+)\end{array}$ & $\begin{array}{c}\Delta Y \\
\text { Cranial-caudal } \\
(+)\end{array}$ & Resultant \\
\hline Liver & -0.7 & -10.0 & 19.5 & 21.9 \\
Spleen & -2.9 & 12.0 & -13.3 & 18.1 \\
R kidney & 15.1 & 3.9 & 17.0 & 23.1 \\
L kidney & 3.0 & 0.5 & -6.2 & 6.9 \\
\hline
\end{tabular}

anterior-posterior direction ( $2.9 \mathrm{~mm}$ posterior). This motion was characterized by a superior and posterior trajectory, essentially in the opposite direction of the liver's motion, but lesser in magnitude. There was a large amount of translation for the right kidney anteriorly $(15.1 \mathrm{~mm})$ and also caudally $(17.0 \mathrm{~mm})$, which was consistent with the liver motion, as they are ipsilateral. Minimal translation was seen for the left kidney in the medial-lateral and anterior-posterior directions with greater translation being seen in the cranial-caudal direction (6.2 mm cranially).

3.3. Morphology Variations. Morphology variations of each abdominal organ in the supine and seated positions were also analyzed qualitatively and quantitatively. Using a best fit alignment method, the model of each organ in the seated position was aligned to its supine counterpart, resulting in an average error difference of $1.9 \mathrm{~mm}$. Surface deviations of each abdominal organ in the seated position were determined relative to the supine position and are shown in Figure 7. The largest deviations from the supine position were in the liver with surface variations ranging from $14.6 \mathrm{~mm}$ to $-12.4 \mathrm{~mm}$. Similar deviations were seen in the spleen and right kidney with variations from $7.2 \mathrm{~mm}$ to $-8.7 \mathrm{~mm}$ and from $8.8 \mathrm{~mm}$ to $-7.9 \mathrm{~mm}$. The smallest deviation was seen in the left kidney with variations from the supine being $6.5 \mathrm{~mm}$ to $-6.1 \mathrm{~mm}$ (Figure 7). Through bounding box measurements, the liver was found to expand $7.8 \%$ in the cranial-caudal direction and compress $5.2 \%$ and $3.4 \%$ in the medial-lateral and anteriorposterior directions. The spleen and left kidney were found to expand little in the anterior-posterior direction $(0.1 \%$ and $1.9 \%$, resp.) while compressing $5.5 \%$ and $6.7 \%$ in the cranial-caudal direction and $2.8 \%$ and $4.1 \%$ in the mediallateral direction. The right kidney was expanded $1.4 \%$ in the 


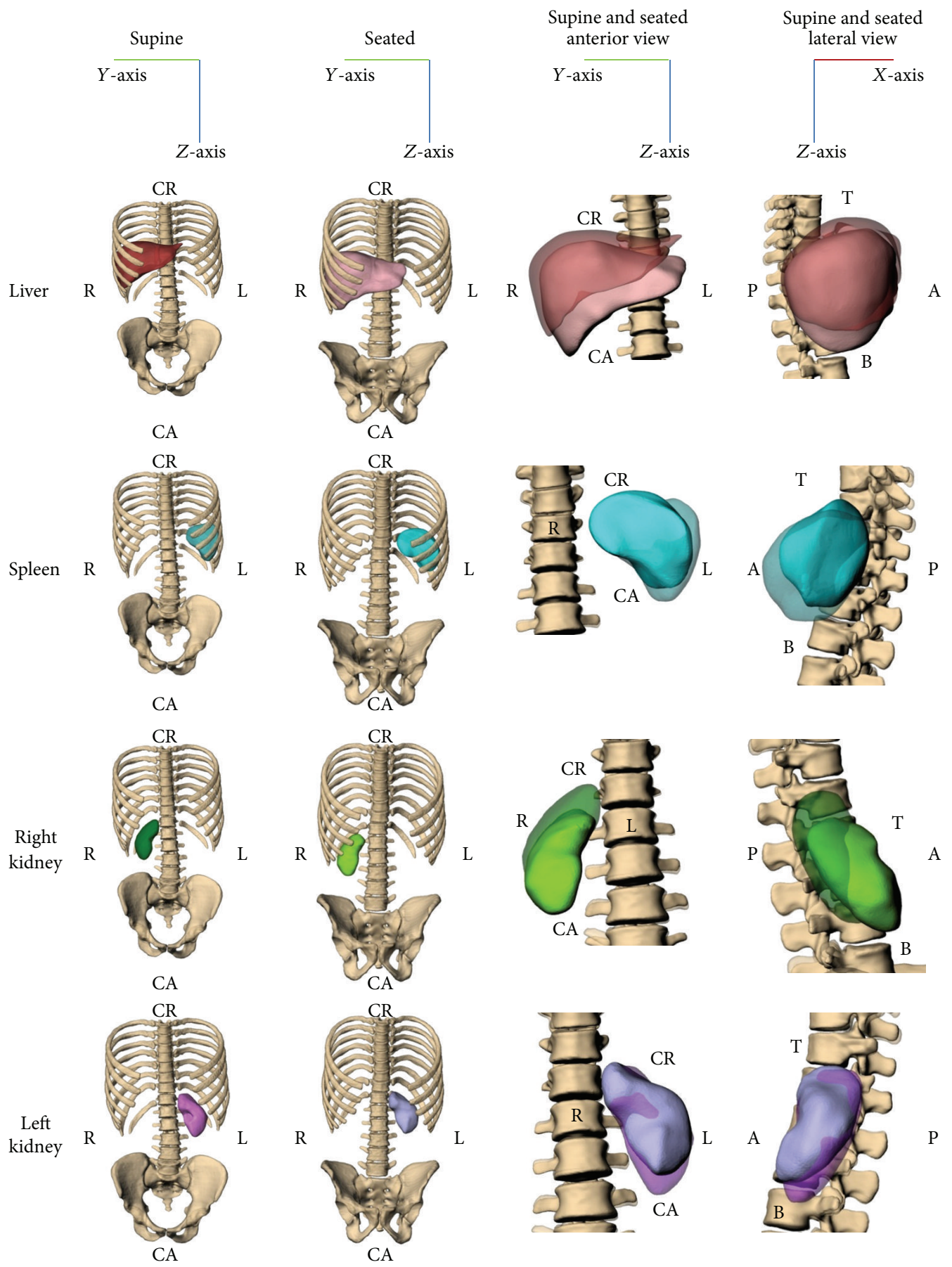

FIGURE 6: Postural changes of M50 abdominal organs with T11 through L2 alignment. Note that the supine organs are transparent in the right two columns, and the relative axis is presented at the top of each column.

medial-lateral direction and compressed $4.6 \%$ and $2.5 \%$ in the cranial-caudal and anterior-posterior directions (Figure 8).

\section{Discussion}

Supine and seated scans from a prospectively scanned midsized male in multiple modalities were investigated to analyze abdominal organ position and morphology between the two postures. The male subject recruited for this study was targeted to match 50th percentile male literature values for height, weight, and 15 additional anthropometric measurements. The goal of this study was to analyze the differences in organ location, morphology, and rib coverage observed due to posture change. The results show that postural changes do affect abdominal organ location, morphology, and projected rib coverage. 


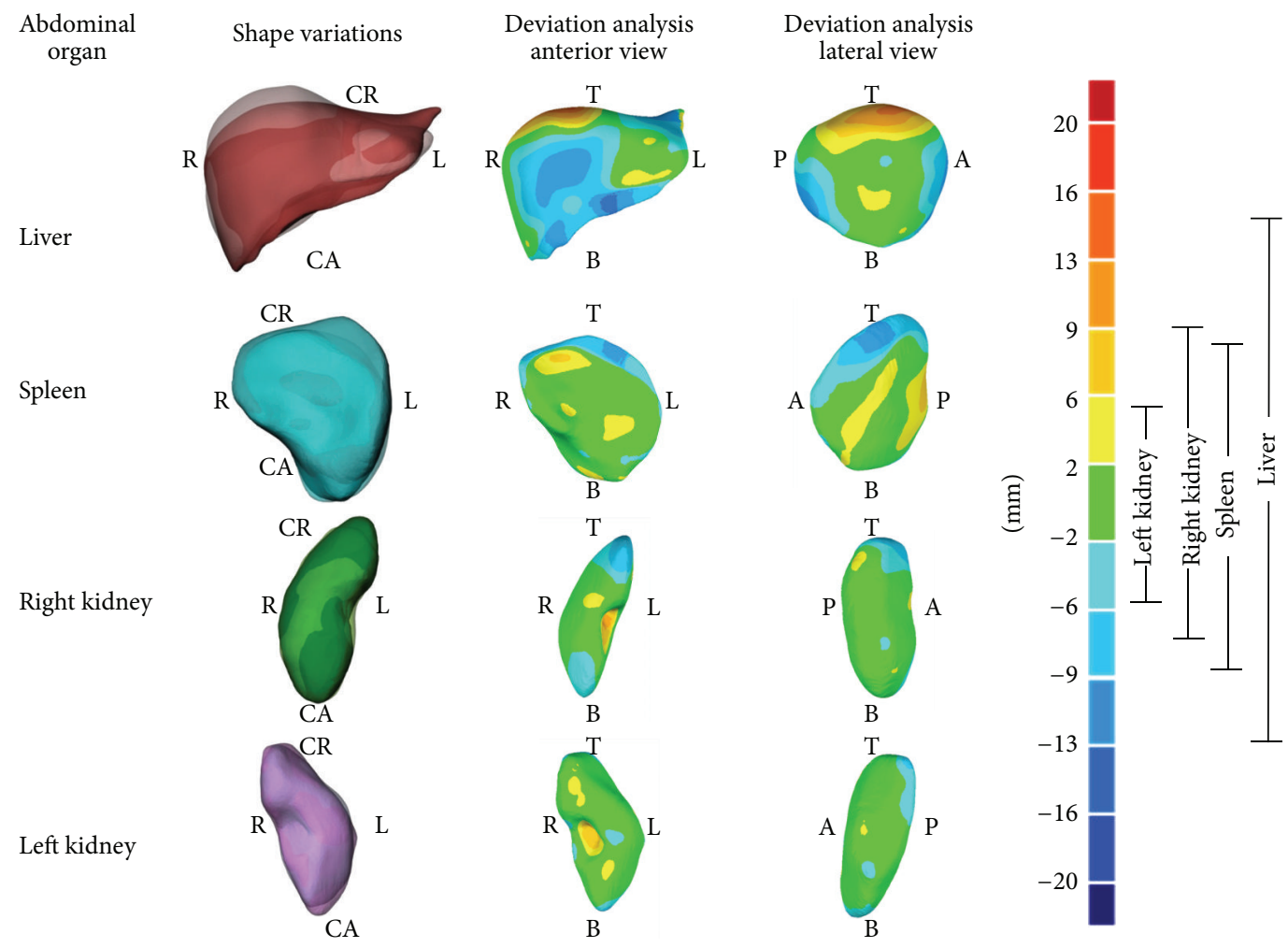

FIGURE 7: Shape variation of abdominal organs in supine position in comparison with seated position (transparent) (left column). Middle and right columns show surface deviation analysis between abdominal organs with the reference shape taken as the organ in the supine scans. Deviations from the reference shown per the color map (right). Positive values indicate an expansion (red) in the region, while negative values indicate a compression (blue) in the region.

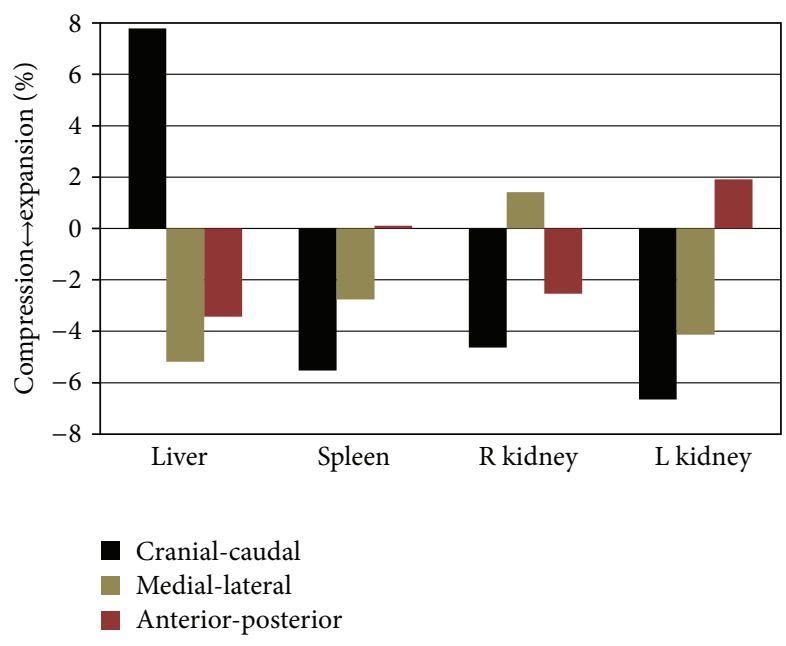

Figure 8: The percent compression (-\%) and expansion (+\%) when transitioning from the supine to seated position.

For the liver, translation of the CG occurred mostly in the cranial-caudal direction, with smaller translation in the anterior-posterior and medial-lateral directions. Relative to the established local coordinate system, its location in the seated posture was inferior to the supine posture; however, the exposed surface was actually decreased due to relative movement of the ribs (Figure 6). Bounded by the chest wall, the spleen translated approximately equal distances along the medial-lateral and cranial-caudal directions $(12.0 \mathrm{~mm}$ leftward and $13.3 \mathrm{~mm}$ cranially, that is, upward) between postures. For the right kidney, translation was minimal in the medial-lateral directions, but large translations were seen in the anterior-posterior direction $(15.1 \mathrm{~mm})$ and cranial-caudal direction $(17 \mathrm{~mm})$. Lastly, the left kidney had little translation in all three directions.

Due to translation and morphology changes of the abdominal organs from the supine and seated positions, rib coverage of organs varies. The study design enabled direct quantification of rib coverage due to the availability of CT scan data. The liver in the frontal and lateral views is protected by the ribs with a $4.6 \%$ (frontal) and $9.6 \%$ (lateral) greater area of coverage in the seated position as compared to the supine position. From a blunt injury perspective these findings are relevant, as the ribs are generally a common load path in blunt force trauma [30]. When viewed laterally, the liver in the seated position is elongated in the cranial-caudal direction, contributing to a greater area of rib coverage. From a frontal view, rib coverage area is larger for the anterior portion of the liver in the seated position. These results have implications for 
computer models whose aim is predicting abdominal injury in an omnidirectional sense. For individuals donning a 3point safety belt over the left shoulder, the liver is located beneath the travel of the belt, and in frontal impacts it is loaded by the belt. In lateral impacts, the studies have shown that laceration of the liver is seen to result from right lateral impacts resulting in rib fractures $[5,31]$.

The spleen is covered by the ribs in both the supine and seated positions, but due to spleen translation when transitioning between each posture, the area of rib coverage is found to increase $11.7 \%$ in the seated position, possibly leading to different injury responses. When examining the right kidney in the supine position, there is rib coverage by the 12th rib resulting in a larger area of rib coverage when compared to the kidney in the seated position where rib coverage declines $12.2 \%$. The left kidney in the supine and seated positions has comparable rib coverage and minimal differences, $0.4 \%$, in exposure between postures. Due to their deep position, the kidneys are less frequently injured in blunt force trauma [2]; however, the rib coverage was conducted on these organs for completeness of the study. The average rib coverage differed between the supine and seated positions, with coverage being less for the kidneys and greater for the liver and spleen in the seated position. From these findings, changes in rib coverage due to posture variations should be considered when using computer models to predict injury.

Rib proximity has noteworthy implications in automobile crashes, as abdominal injury is often associated with rib fracture. As a result of rib coverage and location, organ injury may be more likely due to increased or decreased exposure. Rouhana states that the ribs do provide some protection for abdominal organs, but when the ribs fail due to impact, the protection is eliminated, and injury to these organs is likely [32]. The literature suggests that ribs are likely to play a role in damaging abdominal organs such as the liver, spleen, and kidneys [3]. Additionally, Siegel et al. reported that the spleen is the most common abdominal organ injured in left lateral impacts as a result of blunt impact to the ribs [30]. Based on the findings in this study and also the findings reported in the literature, the area of rib coverage is found to change in various postures, and impact to the ribs can result in injury to the abdominal organs, so this should be considered when studying abdominal injury mechanism.

Morphology variation of the abdominal organs between the supine and seated positions can be readily visualized (Figure 7). Through the completion of a deviation analysis these differences can be quantitatively identified. The greatest deviation in morphology was seen in the liver where the seated abdominal organ varies from the supine abdominal organ by expanding $7.8 \%$ in the cranial-caudal direction and compressing $5.2 \%$ and $3.4 \%$ in the medial-lateral and anterior-posterior directions. The spleen, left kidney, and right kidney in the seated position were also seen to vary in morphology when comparing to the supine position. These findings are consistent with what is known about the relative stiffness of these organs. Material properties of these organs found in the literature show that the spleen on average has $30 \%$ greater elastic modulus than the kidneys and $60 \%$ greater elastic modulus than the liver [33]. Therefore as expected the softer organs are demonstrating more morphological changes from one posture to the other.

The location of abdominal organs and morphology variance due to postural position may be a factor in vehicle crash injuries. Rouhana and Foster reported that many abdominal injuries result from interior vehicle components [34], such as loads from the seat belt or steering wheel. Based on the location and morphology of abdominal organs and the load that is applied to these organs, the severity of the injury may vary. For example, liver injuries are found to increase fivefold when the impact location was lateral rather than frontal. The same was also seen for the kidneys, except the increase in injury was twofold [32-35]. While the most straightforward application of this work is through the development of computational models, it is clear that other types of physical human surrogates for testing could be affected by these findings. Data from this study may also inform the development of future biomechanical experiments studying abdominal injury. In the case of a physical human surrogate model for ballistic impact assessment [7], an understanding of relative organ location should be known to test organ specific injury criteria and accurately define injury mechanisms.

A limitation of this study is that results are specific to an individual representing the 50th percentile male in terms of height, weight, and 15 anthropometric measurements. Due to the very labor intensive data collection (3 imaging modalities of full body data, over 15,000 individual images collected over a 2 month period, while the subject was enrolled in the study), larger sample sizes were not feasible at the time of acquisition. Although these findings are based on one individual and are not scaled, the results indicate that postural changes affect location, morphology, and rib coverage of the liver, spleen, and kidneys.

An analysis of the reported mean volumes for the organs of interest in several literature studies [20,36-42] shows a weighted average mean (taking into account sample size in each study) of $1575 \mathrm{~cm}^{3}, 186 \mathrm{~cm}^{3}, 163 \mathrm{~cm}^{3}$, and $164 \mathrm{~cm}^{3}$ for the liver, spleen, right kidney, and left kidney. By contrast, the subject in this work had volumes of $1255 \mathrm{~cm}^{3}, 205 \mathrm{~cm}^{3}$, $127 \mathrm{~cm}^{3}$, and $141 \mathrm{~cm}^{3}$ for the liver, spleen, right kidney, and left kidney. While there are discrepancies, particularly in the liver, three main points should be noted. The image sets used in the papers referenced previously to examine organ volume all used image acquisition with a much greater slice thickness ( $5 \mathrm{~mm}$ to $20 \mathrm{~mm}$ ) than that which is used in this study (CT: $0.63 \mathrm{~mm}$ and MRI and uMRI: $2 \mathrm{~mm}$ ). In all cases referenced, the slice thickness was at least 2 times as great, with most cases being greater than 5 times larger. Therefore, it is unknown the extent to which the volume differences are based on the effect of lower resolution data. Secondly, the sheer volume of the data required for this study (described previously) made population-based study impractical. Lastly, the goal of this study was to present the methods to perform this analysis on a single subject. The approach for this work was not to scale the organ volumes in any way in order to maximize the true representation of organ proximity to bony structures, location changes, and morphology changes. 
Further studies will be required to determine if these location, morphology, and rib coverage variations are consistent within a wider population of individuals representing the 50th percentile male or in other anthropometrically distinct populations (i.e., 5th percentile small female, 95th percentile large individuals). Two studies examining morphology changes of the organs of interest in the present work can be found in the literature. Chen and Shapiro [43] proposed a method to analyze shape variation based on principle component analysis, and Lamecker et al. [44] proposed a shape analysis method based on minimizing distortion between two surfaces. Both of these studies present methods for shape analysis but do not present results on how shape variation manifests across a population. Studies by Gayzik et al., Danelson et al., Weaver et al., and Urban et al. examine morphology changes in various anatomical structures using Procrustes Analysis [45-48], but none of these are focused on the abdominal organs examined in this study. Furthermore, with adequate data the methods presented here could be used to study vulnerable populations such as the elderly or obese. Researchers have shown that the morphology of the entire rib cage changes with age $[45,49]$.

This study suggests that posture-dependent changes in abdominal organs should be accounted for in the development of human body models for biomechanical research. In the absence of additional data, the results of the study could be used to approximate the effects of gravity on posture. This approach should be used only with caution and as a general guideline due to the limited sample; however, it should be noted that the data was collected prospectively on a thoroughly prescreened, living individual. The medical images were found to be negative for pathology or anatomical abnormalities by a board certified radiologist.

The results indicate that model development based solely on image data in the supine posture will omit changes in the relative location of the key structures of interest. As human body modeling for injury prediction becomes more advanced, it will be important to acquire source data in postures as close to the final posture of interest possible, as this will maximize the morphological accuracy of the models. While comparative studies of models developed with and without posture-dependent data are lacking, based solely on positional changes alone, it is likely that using posturedependent data would have an effect on the injuries predicted in a given simulation.

\section{Conclusion}

A manual and semiautomated image segmentation approach and registration method were used to complete a comparative analysis between abdominal organs in the supine and seated position for an individual representing the 50th percentile male. The findings indicate that variations in organ location, morphology, and proximity to bony structures based on postural changes should be accounted for when constructing finite element models for vehicle safety research. The liver, spleen, right kidney, and left kidney were found to have a resultant translation of $21.9 \mathrm{~mm}, 18.1 \mathrm{~mm}, 23.1 \mathrm{~mm}$, and $6.9 \mathrm{~mm}$ when transitioning from the supine to seated position. Rib coverage was found to increase for the liver and spleen between the supine and seated positions but was found to decrease for the kidneys. For the liver, rib coverage increased from $4.6 \%$ (frontal) to $9.6 \%$ (lateral), and for the spleen organ exposure increased $11.7 \%$ from the supine to seated position. The right and left kidneys had a decrease in coverage of $12.2 \%$ and $0.4 \%$. When examining the surface of the liver, spleen, and kidneys in supine position compared to the seated position, each organ was found to compress or expand in the cranial-caudal, medial-lateral, and anteriorposterior directions. The results from this study provide evidence that organ location, exposure, and morphology are affected by postural changes, which is important in prediction injury mechanisms. Via computational models that accurately reflect organ position and morphology changes with posture, this work will lead to increased knowledge of how blunt injury is influenced by location and morphology variations.

\section{Acknowledgments}

This study was supported by the Global Human Body Models Consortium (WFU-001). Thanks are due to Lucas Swenson who supported the project through the undergraduate work study program at Wake Forest University.

\section{References}

[1] National Highway Traffic Safety Administration, Traffic Safety Facts 2010: A Compilation of Motor Vehicle Crash Data from the Fatality Analysis Reporting System and the General Estimates System, National Center for Statistics and Analysis. U.S. Department of Transportation, Washington, DC, USA, 2010, DOT HS 811659.

[2] K. D. Klinich, C. A. C. Flannagan, K. Nicholson, L. W. Schneider, and J. D. Rupp, Injury in Motor-Vehicle Crashes, University of Michigan Transportation Research Institute, November 2008, UMTRI-2009-40.

[3] C. J. Anders, “Abdominal injuries.," Postgraduate Medical Journal, vol. 43, no. 503, pp. 582-586, 1967.

[4] S. Park, "Clinical analysis for the correlation of intra-abdominal organ injury in the patients with rib fracture," The Korean Journal of Thoracic and Cardiovascular Surgery, vol. 45, no. 4, pp. 246-250, 2012.

[5] A. Al-Hassani, H. Abdulrahman, I. Afifi et al., "Rib fracture patterns predict thoracic chest wall and abdominal solid organ injury," The American Surgeon, vol. 76, no. 8, pp. 888-891, 2010.

[6] E. Shweiki, J. Klena, G. C. Wood, and M. Indeck, "Assessing the true risk of abdominal solid organ injury in hospitalized rib fracture patients," The Journal of Trauma, vol. 50, no. 4, pp. 684688, 2001.

[7] J. C. Roberts, P. J. Biermann, J. V. O'Connor et al., "Modeling nonpenetrating ballistic impact on a human torso," Johns Hopkins APL Technical Digest, vol. 26, no. 1, pp. 84-91, 2005.

[8] J. H. Stuhmiller, Blast Injury: Translating Research into Operation Medicine, Office of the Surgeon General at TMM Publications, Borden Institute, Walter Reed Army Medical Center, Bethesda, Md, USA, 2008, edited by. W. F. Santee and K. E. Friedl. 
[9] Y. Huang, A. I. King, and J. M. Cavanaugh, "Finite element modeling of gross motion of human cadavers in side impact," Stapp Car Crash Journal, vol. 38, pp. 35-53, 1994.

[10] M. Iwamoto, Y. Kisanuki, I. Wantanabe, K. Furusu, K. Miki, and J. Hasegawa, "Development of a finite element model of the Total human Model for Safety (THUMS) and application to injury reconstruction," in International Research Council on the Biomechanics of Injury (IRCOBI '02), Munich, Germany, September 2002.

[11] J. Ruan, R. El-Jawahri, L. Chai, S. Barbat, and P. Prasad, "Prediction and analysis of human thoracic impact responses and injuries in cadaver impacts using a full human body finite element model," Stapp Car Crash Journal, vol. 47, pp. 299-321, 2003.

[12] J. S. Ruan, R. El-Jawahri, S. Barbat, and P. Prasad, "Biomechanical analysis of human abdominal impact responses and injuries through finite element simulations of a full human body model," Stapp Car Crash Journal, vol. 49, pp. 343-366, 2005.

[13] C. S. Shah, K. H. Yang, W. Hardy, H. K. Wang, and A. I. King, "Development of a computer model to predict aortic rupture due to impact loading," Stapp Car Crash Journal, vol. 45, pp. 161182, 2001.

[14] K. Shigeta, Y. Kitagawa, and T. Yasuki, "Development of next generation human FE model capable of organ injury prediction," in Proceedings of the 21st Annual Enhanced Safety of Vehicles, Stuttgart, Germany, June 2009.

[15] K. H. Yang, J. Hu, N. A. White, A. I. King, C. C. Chou, and P. Prasad, "Development of numerical models for injury biomechanics research: a review of 50 years of publications in the Stapp Car Crash Conference," Stapp Car Crash Journal, vol. 50, pp. 429-490, 2006.

[16] S. Robin, "Human model for safety-a joint effort towards the development of redefined human-like car-occupant models," in Proceedings of the 17th International Conference for the Enhanced Safety of Vehicles, Amsterdam, The Netherlands, June 2001.

[17] J. S. Ruan, R. El-Jawahri, S. W. Rouhana, S. Barbat, and P. Prasad, "Analysis and evaluation of the biofidelity of the human body finite element model in lateral impact simulations according to ISO-TR9790 procedures," Stapp Car Crash Journal, vol. 50, pp. 491-507, 2006.

[18] J. C. Roberts, A. C. Merkle, P. J. Biermann et al., "Computational and experimental models of the human torso for nonpenetrating ballistic impact," Journal of Biomechanics, vol. 40, no. 1, pp. 125-136, 2007.

[19] J. C. Roberts, T. P. Harrigan, E. E. Ward, T. M. Taylor, M. S. Annett, and A. C. Merkle, "Human head-neck computational model for assessing blast injury," Journal of Biomechanics, vol. 45, no. 16, pp. 2899-2906, 2012.

[20] P. Beillas, Y. Lafon, and F. W. Smith, "The effects of posture and subject-to-subject variations on the position, shape and volume of abdominal and thoracic organs," Stapp Car Crash Journal, vol. 53, pp. 127-154, 2009.

[21] Y. Lafon, F. W. Smith, and P. Beillas, "Combination of a modeldeformation method and a positional MRI to quantify the effects of posture on the anatomical structures of the trunk," Journal of Biomechanics, vol. 43, no. 7, pp. 1269-1278, 2010.

[22] F. Gayzik, C. Hamilton, J. Tan et al., "A multi-modality image data collection protocol for full body finite element model development," SAE Technical Paper 2009-01-2261, June 2009.

[23] F. S. Gayzik, D. P. Moreno, C. P. Geer, S. D. Wuertzer, R. S. Martin, and J. D. Stitzel, "Development of a full body
CAD dataset for computational modeling: a multi-modality approach," Annals of Biomedical Engineering, vol. 39, no. 10, pp. 2568-2583, 2011.

[24] M. P. Reed, M. A. Manary, and L. W. Schneider, "Methods for measuring and representing automobile occupant posture," SAE Technical Paper 1999-01-0959, March 1999.

[25] F. S. Gayzik, D. P. Moreno, K. A. Danelson, C. McNally, K. D. Klinich, and J. D. Stitzel, "External landmark, body surface, and volume data of a mid-sized male in seated and standing postures," Annals of Biomedical Engineering, vol. 40, no. 9, pp. 2019-2032, 2012.

[26] C. Gordon, T. Churchill, C. Clauser et al., "Anthropometric survey of U.S.army personnel: methods and summary statistics 1988," Tech. Rep. Natick/TR-89/044, Prepared for United States Army Natick Research, Development, and Engineering Center, Natick, Mass, USA, 1989.

[27] C. A. C. Flannagan, M. A. Manary, L. W. Schneider, and M. P. Reed, "Improved seating accommodation model with application to different user populations," in Proceedings of the SAE International Congress \& Exposition, pp. 43-50, February 1998.

[28] G. Wu, F. C. T. van der Helm, H. E. J. Veeger et al., "ISB recommendation on definitions of joint coordinate systems of various joints for the reporting of human joint motion-part II: shoulder, elbow, wrist and hand," Journal of Biomechanics, vol. 38, no. 5, pp. 981-992, 2005.

[29] Society of Automotive Engineers, "J1733: Sign Convention for Vehicle Crash Testing," SAE Standards 2007.

[30] J. H. Siegel, S. Mason-Gonzalez, P. Dischinger et al., "Safety belt restraints and compartment intrusions in frontal and lateral motor vehicle crashes: mechanisms of injuries, complications, and acute care costs," The Journal of Trauma, vol. 34, no. 5, pp. 736-759, 1993.

[31] D. C. Viano, I. V. Lau, D. V. Andrzejak, and C. Asbury, "Biomechanics of injury in lateral impacts," Accident Analysis and Prevention, vol. 21, no. 6, pp. 535-551, 1989.

[32] S. W. Rouhana, Biomechanics of Abdominal Trauma, Springer Science and Business Media, New York, NY, USA, 1993.

[33] J. B. Lee and K. H. Yang, "Development of a finite element model of the human abdomen," Stapp Car Crash Journal, vol. 45, pp. 79-100, 2001.

[34] S. W. Rouhana and M. E. Foster, "Later impact-an analysis of the statistics in the NCSS," in Proceedings of the 29th Stapp Car Crash Conference, pp. 79-98, Washington, DC, USA, 1985.

[35] C. F. Baxter and R. D. Williams, "Blunt abdominal trauma," The Journal of Trauma, vol. 1, pp. 241-247, 1961.

[36] X. P. Zhou, T. Lu, Y. G. Wei, and X. Z. Chen, "Liver volume variation in patients with virus-induced cirrhosis: findings on MDCT,' American Journal of Roentgenology, vol. 189, no. 3, pp. W153-159, 2007.

[37] E. M. Geraghty, J. M. Boone, J. P. McGahan, and K. Jain, "Normal organ volume assessment from abdominal CT," Abdominal Imaging, vol. 29, no. 4, pp. 482-490, 2004.

[38] J. M. Henderson, S. B. Heymsfield, J. Horowitz, and M. H. Kutner, "Measurement of liver and spleen volume by computed tomography. Assessment of reproducibility and changes found following a selective distal splenorenal shunt," Radiology, vol. 141, no. 2, pp. 525-527, 1981.

[39] J. Solvig, H. Ekberg, F. Hansen, J. Brunkvall, and T. Länne, "Accuracy of noninvasive ultrasonic volume measurements on human kidney transplants. Presentation of a new formula," Nephron, vol. 80, no. 2, pp. 188-193, 1998. 
[40] P. Prassopoulos, M. Daskalogiannaki, M. Raissaki, A. Hatjidakis, and N. Gourtsoyiannis, "Determination of normal splenic volume on computed tomography in relation to age, gender and body habitus," European Radiology, vol. 7, no. 2, pp. 246-248, 1997.

[41] L. O. Lerman, A. L. Flickinger, P. F. Sheedy, and S. T. Turner, "Reproducibility of human kidney perfusion and volume determinations with electron beam computed tomography," Investigative Radiology, vol. 31, no. 4, pp. 204-210, 1996.

[42] G. L. de la Grandmaison, I. Clairand, and M. Durigon, "Organ weight in 684 adult autopsies: new tables for a Caucasoid population," Forensic Science International, vol. 119, no. 2, pp. 149-154, 2001.

[43] J. H. Chen and L. G. Shapiro, "3D point correspondence by minimum description length with 2DPCA," in Proceedings of Annual International Conference of the IEEE Engineering in Medicine and Biology Society, pp. 5657-5660, 2009.

[44] H. Lamecker, T. Lange, and M. Seebass, "A statistical shape model for the liver," in Proceedings of the 5th International Conference on Medical Image Computing and Computer-Assisted Intervention-Part II, Tokyo, Japan, September 2002.

[45] F. S. Gayzik, M. M. Yu, K. A. Danelson, D. E. Slice, and J. D. Stitzel, "Quantification of age-related shape change of the human rib cage through geometric morphometrics," Journal of Biomechanics, vol. 41, no. 7, pp. 1545-1554, 2008.

[46] J. E. Urban, J. A. Maldjian, C. T. Whitlow, and J. D. Stitzel, “A method to investigate the size and shape variation of the lateral ventricles with age," Biomedical Sciences Instrumentation, vol. 48, pp. 447-453, 2012.

[47] K. A. Danelson, C. P. Geer, J. D. Stitzel, D. E. Slice, and E. G. Takhounts, "Age and gender based biomechanical shape and size analysis of the pediatric brain," Stapp Car Crash Journal, vol. 52, pp. 59-81, 2008.

[48] A. A. Weaver, E. A. Moody, E. G. Armstrong, and J. D. Stitzel, "Image segmentation and registration algorithm to collect homologous landmarks for age-related thoracic morphometric analysis," Biomedical Sciences Instrumentation, vol. 47, pp. 7076, 2011.

[49] R. Kent, S. H. Lee, K. Darvish et al., "Structural and material changes in the aging thorax and their role in crash protection for older occupants," Stapp Car Crash Journal, vol. 49, pp. 231249, 2005. 


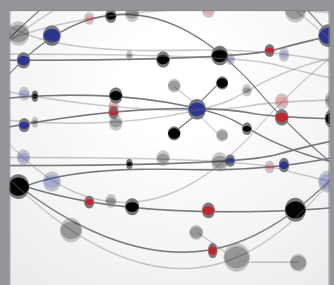

The Scientific World Journal
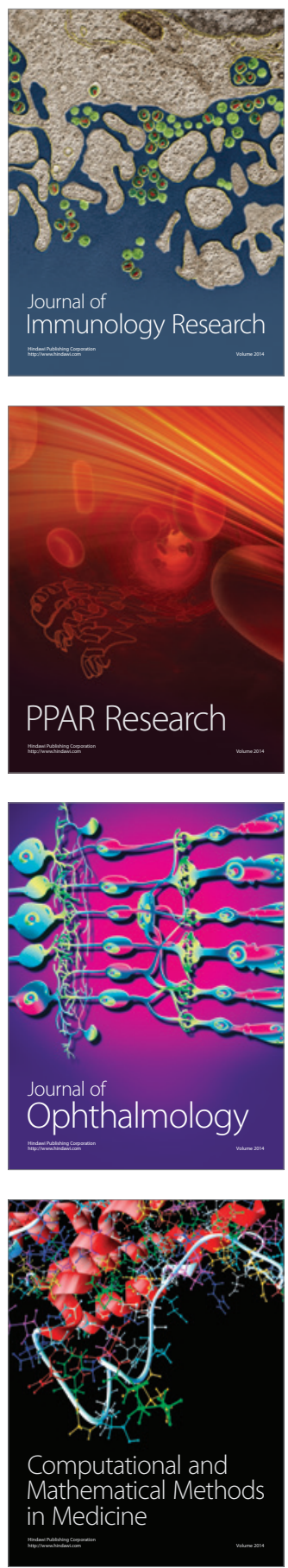

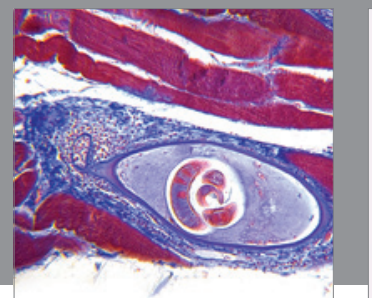

Gastroenterology

Research and Practice
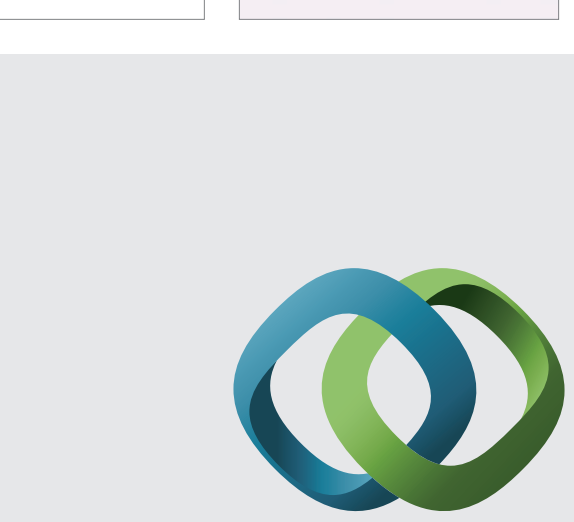

\section{Hindawi}

Submit your manuscripts at

http://www.hindawi.com
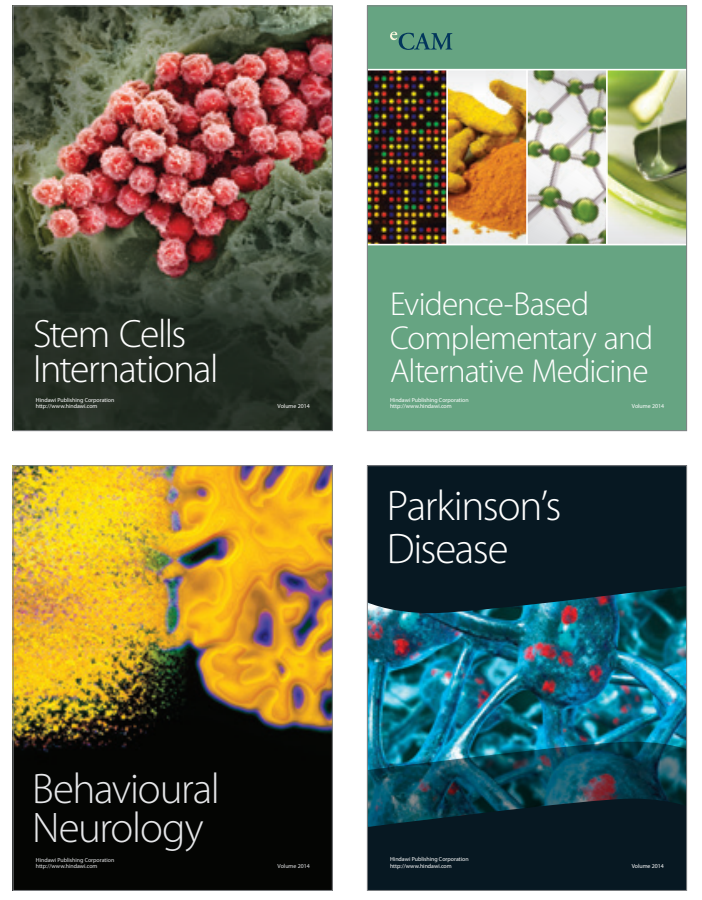
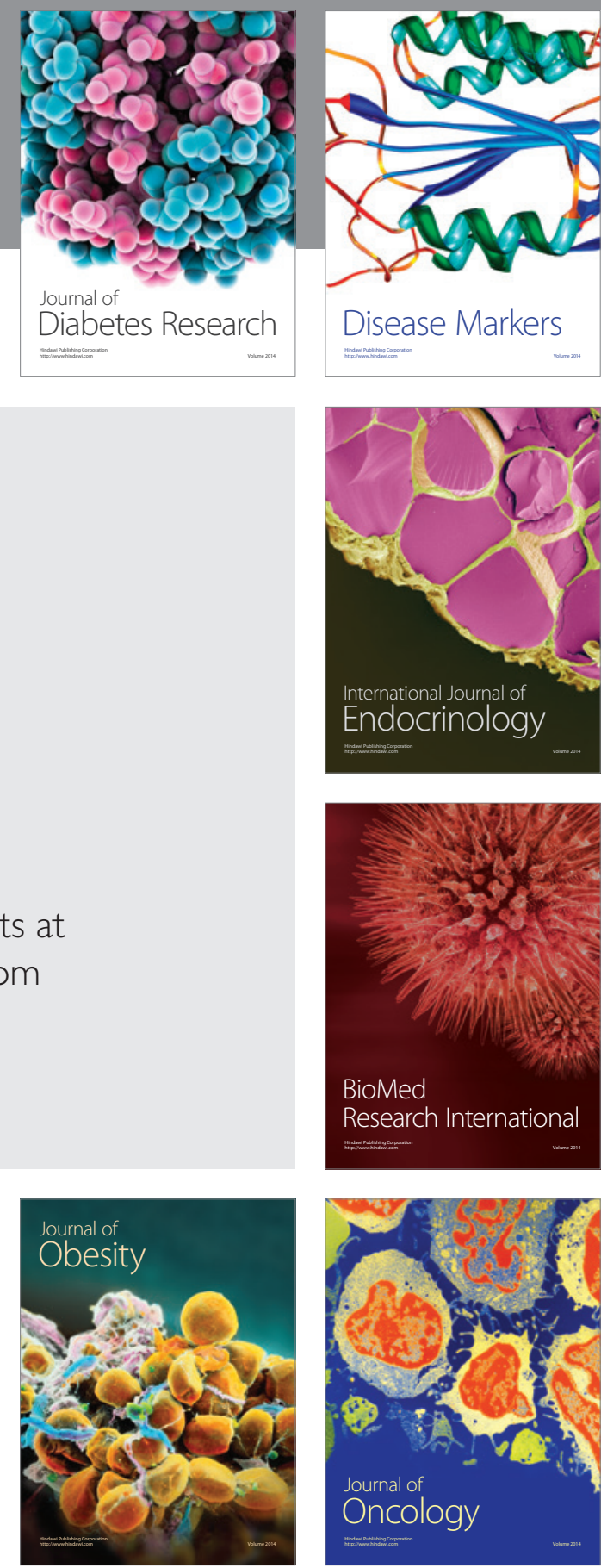

Disease Markers
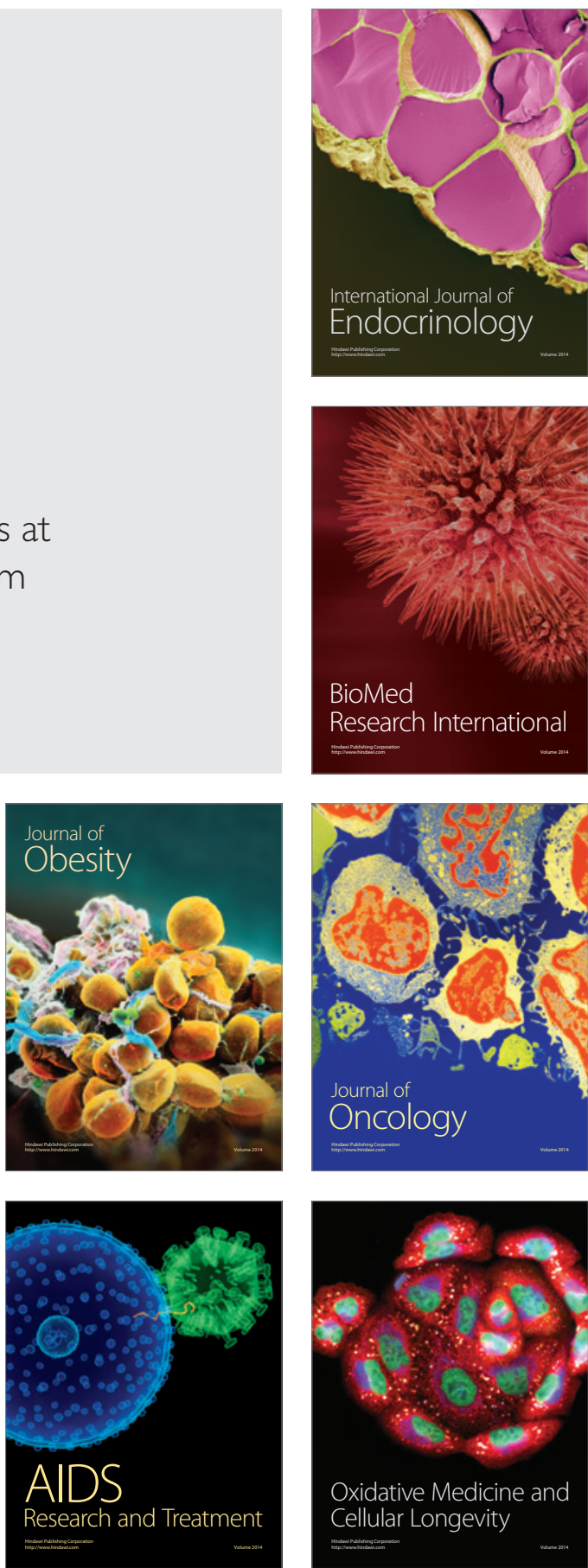